\title{
Effects of Chronic Glucocorticoid Receptor Stimulation on Circadian Locomotor Activity and Serotonergic Neurotransmission in the Basolateral Amygdala of Rats
}

\author{
Norifumi Shimizu* and Chiaki Hara \\ Center for Advancing Pharmaceutical Education, Daiichi University of Pharmacy; 22-1 Tamagawa-cho, Minami-ku, \\ Fukuoka 815-8511, Japan.
}

Received March 12, 2020; accepted June 2, 2020

Clinical studies, especially those in animal models, have provided evidence that chronic stress may play a role in the etiology of psychiatric diseases, such as depression. Because chronic stress activates the hypothalamic-pituitary-adrenal (HPA) axis, resulting in the excessive secretion of glucocorticoids, the chronic stimulation of glucocorticoid receptors (GRs) may be involved in the pathogenesis of depression. To further investigate the relationship between GR activation and depression, we used the synthetic glucocorticoid dexamethasone (DEX) and the GR antagonist mifepristone to examine the effects of chronic GR stimulation on the circadian rhythms of locomotor activity and serotonergic neurotransmission in the basolateral amygdala (BLA) of rats. Chronic treatment with DEX reduced locomotor activity during the dark phase, without changing overall activity patterns. Measuring the basal release of serotonin in the BLA, using in vivo microdialysis, confirmed that chronic treatment with DEX induced serotonergic hypofunction in the BLA. The co-administration of DEX with mifepristone effectively suppressed the depressive-like symptoms caused by chronic treatment with DEX. Our results provided further evidence for a relationship between GR and depression and suggest that the pharmacological blockade of GR may increase the effectiveness of conventional pharmacotherapies used to treat depression.

Key words depression; glucocorticoid receptor; dexamethasone; mifepristone; circadian locomotor activity; serotonergic neurotransmission

\section{INTRODUCTION}

Many studies have examined the effects of antidepressants, using paradigms designed to mimic the symptom of human depression. ${ }^{1,2)}$ Because the hypothalamic-pituitary-adrenal (HPA) axis is well-known to be involved in the pathophysiology of depression in humans, ${ }^{3)}$ chronic treatment with corticosterone, a major glucocorticoid in rodents, has been used to generate an animal model of depression. ${ }^{4,5}$ The effects of corticosterone are mediated by two types of intracellular receptors: glucocorticoid receptors (GRs) and mineralocorticoid receptors. Recently, treatment with the GR antagonist mifepristone has been shown to relieve the symptoms of depression in clinical studies. ${ }^{6}$ In animal models of depression, treatment with mifepristone reversed the observed increase in immobility times during the forced swim test, in a rat maternal separation model. ${ }^{7)}$ Furthermore, a GR antagonist, RU-43044, was able to counteract the repeated-corticosteroneinjection-induced increase in immobility time during the forced swim test and the tail suspension test, in mice. ${ }^{8)}$ These results indicated that the depressive-like symptoms induced by chronic corticosterone treatment were mediated by the chronic stimulation of GRs.

Decreased locomotor activity has often been used as an indicator of depressive-like symptoms, ${ }^{9)}$ and the chronic stimulation of GRs may also be involved in this phenomenon. However, basal locomotor activity is also greatly affected by circadian rhythms; thus, the chronic stimulation of GRs may have differential effects on locomotor activity during light and dark phases.
The central serotonin systems in the brain are well-known to play a crucial role in depression. The mechanisms of most major effective therapeutic agents used to treat depression, such as tricyclic antidepressants (TCAs) and selective serotonin reuptake inhibitors (SSRIs), result in enhanced serotonergic neurotransmission in the brain. However, the relationship between the central serotonin system and GR activity remains unclear.

Therefore, we used dexamethasone (DEX) and mifepristone to investigate the effects of GR activation on circadian locomotor activity and the basal release of serotonin in the basolateral amygdala (BLA) of rats.

\section{MATERIALS AND METHODS}

Animals Male Sprague-Dawley rats ( 8 weeks old at the beginning of the experiment) were obtained from Kyudo Co., Ltd. (Fukuoka, Japan). The rats were maintained under steady condition $\left(24 \pm 1{ }^{\circ} \mathrm{C}\right.$, with a $12 \mathrm{~h}$ light-dark cycle with lights on at 7:00). Our studies were conducted in accordance with the Guide for Care and Use of Laboratory Animals of Daiichi University of Pharmacy, which is accredited by the Ministry of Education, Culture, Sports, and Technology of Japan.

Treatment The rats were divided into 3 groups: 1) the chronic saline treatment in combination with saline group, 2) the chronic DEX treatment in combination with saline group, and 3) the chronic DEX treatment in combination with mifepristone group. In this study, chronic treatment with DEX or saline was administered using an Alzet ${ }^{\circledR}$ osmotic pump (model $2 \mathrm{ML} 2 ; 5 \mu \mathrm{L} / \mathrm{h}$ for $14 \mathrm{~d}$ ). On the first day of chronic 
treatment (day 1), an osmotic pump was implanted subcutaneously into the back of each rat under isoflurane anesthesia. DEX was infused into each rat from the osmotic pump, at a dose of $0.5 \mathrm{mg} / \mathrm{kg} / \mathrm{d}$ for $14 \mathrm{~d}$ (days 1-14). During chronic treatment with DEX, mifepristone $(20 \mathrm{mg} / \mathrm{kg}$, intraperitoneally) or saline was administered to rats, twice a day (at 9:00 and 19:00), until day 13. DEX solution was prepared by diluting the DECADRON ${ }^{\circledR}$ Phosphate Injection (Aspen Japan K.K., Tokyo, Japan) with saline. Mifepristone (Sigma-Aldrich Japan K.K., Tokyo, Japan) was suspended in saline.

Circadian Locomotor Activity On day 11, the rats were habituated to a locomotion counter chamber $(40 \mathrm{~cm} \times 40 \mathrm{~cm}$, EIKO Science Co., Ltd., Tokushima, Japan) for 12 h (day 11, 19:00-day 12, 7:00). Horizontal movement was detected for 24h (light: day 12, 7:00-19:00, dark: day 12, 19:00-day 13, $7: 00)$ by a $16 \times 16$ IR photo-beam array, placed $1.5 \mathrm{~cm}$ above the bottom of the chamber. Throughout habituation and locomotor measurement, food and water were available ad libitum.

Surgery On day 13, immediately after the measurement of locomotor activity, rats were anesthetized with isoflurane and then placed on a stereotaxic apparatus. A guide cannula was implanted just above the BLA (AP: $6.4 \mathrm{~mm}$, ML: $5.0 \mathrm{~mm}$, DV: $5.7 \mathrm{~mm}$, relative to the interaural), as described by Paxinos and Watson (2007).

In Vivo Microdialysis On day 14 , at least $24 \mathrm{~h}$ after surgery, a microdialysis probe was gently inserted through the guide cannula into the BLA. The probe was perfused at a rate of $1 \mu \mathrm{L} / \mathrm{min}$ with artificial cerebrospinal fluid, containing the following electrolytes (in $\mathrm{mM}$ ); $150 \mathrm{Na}^{+}, 3.0 \mathrm{~K}^{+}, 1.4 \mathrm{Ca}^{2+}, 0.8$ $\mathrm{Mg}^{2+}, 1 \mathrm{PO}_{4}^{3-}$, and $155 \mathrm{Cl}^{-}$, at $\mathrm{pH}$ 7.4. A 3-h stabilization period was allowed before the dialysate was collected. Collected fractions of dialysate were analyzed on-line for serotonin contents every $20 \mathrm{~min}$, using a HPLC with electrochemical detection (Eicom Co., Ltd., Kyoto, Japan). The final dialysate collection was completed before 17:00. Serotonin was separated on an Eicompak CA-5ODS (Eicom Co., Ltd.).

Statistical Analysis Data are presented as the mean and the standard error of the mean (S.E.M.). All data were analyzed using a one-way ANOVA, followed by the TukeyKramer test. Differences were considered to be significant when $p$-values were less than 0.05 .

\section{RESULTS}

Circadian Locomotor Activity To evaluate the effects of chronic GR stimulation on circadian locomotor activity, we measured rat locomotor activity for $24 \mathrm{~h}$ (day 12, 7:00-day $13,7: 00)$. In preliminary experiments, we confirmed that the circadian locomotor activity was not affected by the acute administration of mifepristone (Light phase: $\mathrm{F} 3,16=0.75$, $p=0.54$, Dark phase: F3,16 $=0.25, p=0.86$, Table 1 ). Hourly locomotor activity and total activity levels during light and dark phases were measured and averaged within each treatment group. Locomotor activity showed clear circadian rhythms for all groups, with reduced activity levels during the light phase and increased activity levels during the dark phase. Chronic DEX treatment, in combination with either saline or mifepristone, did not affect the total activity level during the light phase $\left(\mathrm{F}_{2,15}=3.13, p=0.07\right.$, Fig. 1). In contrast, the total activity level during the dark phase was significantly altered by chronic DEX treatment $\left(\mathrm{F}_{2,15}=8.26, p<0.01\right.$, Fig. 1). Post hoc analysis indicated that chronic DEX treatment resulted in a significant decrease in the total activity level $(p<0.01)$. However, chronic DEX treatment combined with mifepristone treatment resulted in a total activity level similar to the control level $(p<0.05)$.

Serotoninergic Neuronal Functions in the BLA To evaluate the effects of chronic GR stimulation serotonergic neuronal function, we determined the basal extracellular serotonin levels in the BLA on day 14, using in vivo microdialysis. Chronic DEX treatment significantly affected basal extracellular serotonin levels in the BLA $\left(\mathrm{F}_{2,15}=9.29, p<0.01\right.$, Fig. 2). Post hoc analysis indicated that chronic DEX treatment led to a significant decrease in the basal extracellular serotonin level in the BLA $(p<0.05)$. The effects of chronic DEX treatment could be completely inhibited by the combined administration of DEX with mifepristone $(p<0.01)$.

\section{DISCUSSION}

The circadian oscillator in the suprachiasmatic nucleus ( $\mathrm{SCN}$ ), which is regarded to be the master clock in mammals, is regulated by environmental light/dark cues conveyed from the eye. ${ }^{10)}$ In nocturnal animals, such as rats, the SCN master clock increases wakefulness and locomotor activity during the dark phase and induces sleep during the light phase. The present study showed that chronic treatment with DEX did not alter the circadian rhythms of rats but did reduce locomotor activity during the dark phase. Decreased locomotor activity has often been used as an indicator of depressive-like symptoms, and chronic stress has been reported to decrease locomotor activity. ${ }^{9)}$ In the present study, chronic treatment with DEX also decreased locomotor activity during the dark phase, which could be prevented by the combined administration of DEX with the GR antagonist mifepristone. These results suggested that the chronic-stress-induced decrease in locomotor activity may be due to the chronic stimulation of GR by glucocorticoids secreted during chronic stress. At present, the mechanism through which the chronic stimulation of GR reduces the locomotor activity, only during the dark

Table 1. Effects of Acute Administration of Mifepristone (MIF) on Circadian Locomotor Activity in Rats

\begin{tabular}{lcccc}
\hline \hline & & & \multicolumn{2}{c}{ MIF } \\
\cline { 2 - 5 } & SAL & $5 \mathrm{mg} / \mathrm{kg}$ & $10 \mathrm{mg} / \mathrm{kg}$ & $20 \mathrm{mg} / \mathrm{kg}$ \\
\hline Light phase & $851.6 \pm 92.8$ & $937.2 \pm 111.7$ & $820.0 \pm 84.6$ & $742.8 \pm 70.3$ \\
Dark phase & $3242.4 \pm 380.5$ & $3468.0 \pm 488.6$ & $2955.8 \pm 562.8$ & $2982.4 \pm 485.0$ \\
\hline
\end{tabular}

Each data represents the mean number of beam breaks for $12 \mathrm{~h} \pm$ standard error of the mean (S.E.M.) for 5 animals. The horizontal movement was detected for $24 \mathrm{~h}$ (Light phase: 7:00-19:00, Dark phase: 19:00-7:00). Saline (SAL) or each dose of MIF was administrated intraperitoneally two times: once in the light phase (7:00) and once in the dark phase (19:00). 
Locomotor activity
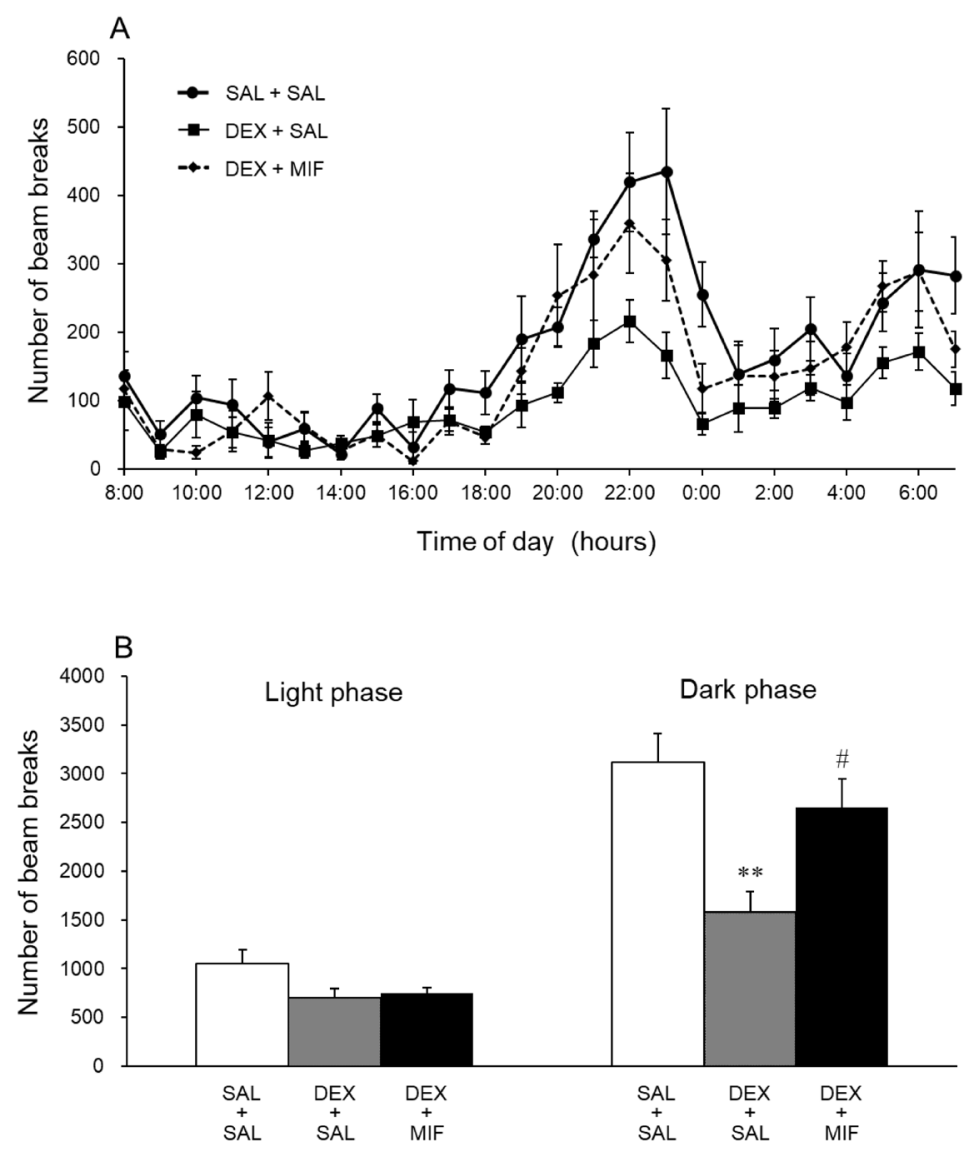

Fig. 1. Effects of Chronic DEX Treatment, in Combination with Either Saline (SAL) or Mifepristone (MIF), on Hourly Locomotor Activity (A) and Total Activity, during Light and Dark Phases (B) in Rats

Ordinate: number of beam breaks. Each column represents the mean number of beam breaks \pm standard error of the mean (S.E.M.) for 6 animals. $* * p<0.01 v s$. chronic saline treatment in combination with saline (SAL $+\mathrm{SAL})$ group. $\# p<0.05 v s$. chronic DEX treatment in combination with saline (DEX $+\mathrm{SAL})$ group.

Basal extracellular serotonin level
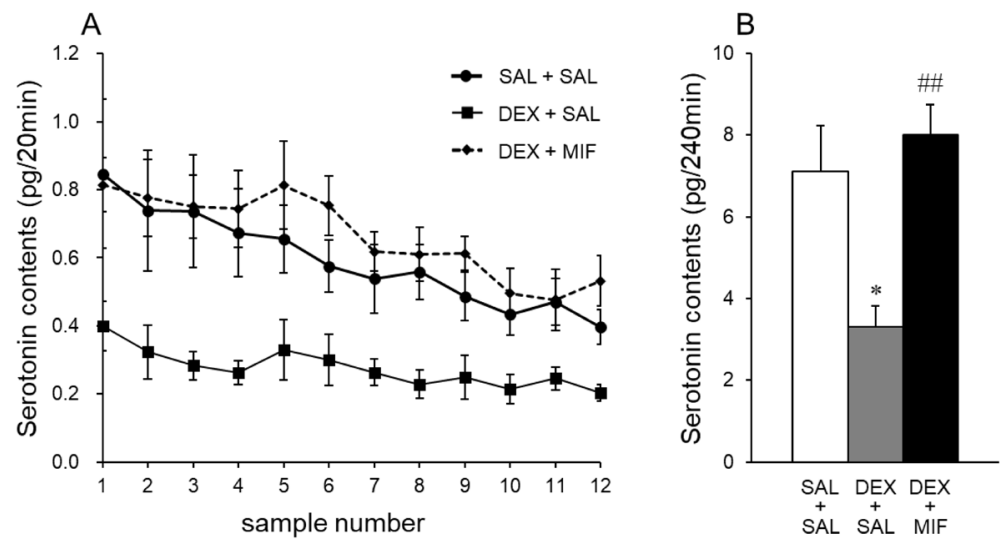

Fig. 2. Effects of Chronic DEX Treatment, in Combination with Either Saline (SAL) or Mifepristone (MIF), on the Basal Extracellular Serotonin Level in the Basolateral Nucleus of the Amygdala

(A) Serotonin contents in a dialysate fraction collected for $20 \mathrm{~min}$. (B) Total serotonin level in the dialysate fraction collected for 240 min. Each column represents the mean amount of serotonin in the dialysate \pm S.E.M. for 6 animals. ${ }^{*} p<0.05 v s$. chronic saline treatment in combination with saline (SAL + SAL) group. \#\#p $<0.01 v s$. chronic DEX treatment in combination with saline (DEX + SAL) group.

phase, is unclear. However, it has been reported that peripherally administered corticosterone stimulates the locomotor activity and the extracellular dopamine levels in the nucleus accumbens, only during the dark phase, and these effects are abolished by the neurochemical (6-hydroxydopamine) deple- tion of the nucleus accumbens dopamine. ${ }^{11)}$ Since dopamine activity is higher in the dark than in the light phase, ${ }^{12)}$ it is possible that chronic GR stimulation causes desensitization of the dopaminergic neural activity, which results in a decreased locomotor activity only during the dark phase. Chronic DEX 
treatment decreased locomotor activity during the dark phase; however, the overall pattern of activity, which peaked at approximately 22:00, then declined before increasing again at approximately 6:00, was not affected. This result suggested that chronic DEX treatment may not affect basic patterns of activity, such as eating and drinking.

The BLA receives serotonergic projections from the dorsal raphe nucleus (DRN), the largest serotonergic nucleus in the brain. ${ }^{13)}$ Thus, the basal release of serotonin in the BLA has been thought to reflect overall serotonergic neural activity in the brain. In the present study, using an in vivo microdialysis technique in freely moving rats, we directly confirmed that chronic treatment with DEX decreased the basal release of serotonin in the BLA. Serotonergic neurons in the DRN have been reported to express GRs, ${ }^{14)}$ and the manipulation of systemic glucocorticoid levels have been shown to regulate the expression and activity of tryptophan hydroxylase 2 (TPH2), the rate-limiting enzyme for serotonin synthesis in the brain. ${ }^{15,16)}$ Taken together with these previous findings, our results suggested that the observed decrease in basal serotonin release in the BLA induced by chronic DEX treatment may be the result of decreased serotonin synthesis, caused by a decrease in TPH2 expression following chronic GR stimulation in the DRN. Since bilateral lesions of serotonergic terminals in the amygdala by 5,7-dihydroxytryptamine did not influence the locomotor activity in rats, ${ }^{17)}$ the decrease in basal serotonin release in the BLA by chronic stimulation of GR, as observed in this study, may not be associated with a decrease in the locomotor activity during the dark phase. It has been reported that elevated levels of serotonin in the hippocampus and prefrontal cortex (PFC) are associated with an increase in the locomotor activity. ${ }^{18)}$ Therefore, chronic GR stimulation may also reduce the serotonin release in the hippocampus and $\mathrm{PFC}$, which may be partly responsible for the decrease in the locomotor activity during the dark phase. Due to the possible involvement of dopaminergic nerves, as mentioned above, we plan to investigate the underlying mechanism by which chronic stimulation of GR reduces the locomotor activity only during the dark phase.

In this study, the GR antagonist mifepristone prevented both the chronic-DEX-induced reduction in locomotor activity during the active phase and serotonergic hypofunction. Although DEX was continuously infused by an osmotic pump, whereas mifepristone was administered twice a day (9:00 and 19:00), the preventive effects of mifepristone were sufficient. Although further investigations will be required to confirm the pharmacological, pharmacokinetic and clinical properties of mifepristone, our present findings suggested that the pharmacological blockade of GR by mifepristone may increase the effectiveness of conventional pharmacotherapies for depression.

Conflict of Interest The authors declare no conflict of interest.

\section{REFERENCES}

1) $\mathrm{Xu}$ Y, Barish PA, Pan J, Ogle WO, O’Donnell JM. Animal models of depression and neuroplasticity: assessing drug action in relation to behavior and neurogenesis. Methods Mol. Biol., 829, 103-124 (2012).

2) Cryan JF, Markou A, Lucki I. Assessing antidepressant activity in rodents: recent developments and future needs. Trends Pharmacol. Sci., 23, 238-245 (2002).

3) Dinan TG. Glucocorticoids and the genesis of depressive illness. A psychobiological model. Br. J. Psychiatry, 164, 365-371 (1994).

4) Gregus A, Wintink AJ, Davis AC, Kalynchuk LE. Effect of repeated corticosterone injections and restraint stress on anxiety and depression-like behavior in male rats. Behav. Brain Res., 156, 105-114 (2005).

5) Johnson SA, Fournier NM, Kalynchuk LE. Effect of different doses of corticosterone on depression-like behavior and HPA axis responses to a novel stressor. Behav. Brain Res., 168, 280-288 (2006).

6) Block TS, Kushner H, Kalin N, Nelson C, Belanoff J, Schatzberg A. Combined analysis of mifepristone for psychotic depression: plasma levels associated with clinical response. Biol. Psychiatry, 84, 46-54 (2018).

7) Iijima $\mathrm{M}$, Ito A, Kurosu S, Chaki S. Pharmacological characterization of repeated corticosterone injection-induced depression model in rats. Brain Res., 1359, 75-80 (2010).

8) Ago Y, Arikawa S, Yata M, Yano K, Abe M, Takuma K, Matsuda T. Antidepressant-like effects of the glucocorticoid receptor antagonist RU-43044 are associated with changes in prefrontal dopamine in mouse models of depression. Neuropharmacology, 55, 1355-1363 (2008).

9) Li R, Wang X, Qin T, Qu R, Ma S. Apigenin ameliorates chronic mild stress-induced depressive behavior by inhibiting interleukin-1 $\beta$ production and NLRP3 inflammasome activation in the rat brain. Behav. Brain Res., 296, 318-325 (2016).

10) Lowrey PL, Takahashi JS. Mammalian circadian biology: elucidating genome-wide levels of temporal organization. Annu. Rev. Genomics Hum. Genet., 5, 407-441 (2004).

11) Piazza PV, Rougé-Pont F, Deroche V, Maccari S, Simon H, Le Moal M. Glucocorticoids have state-dependent stimulant effects on the mesencephalic dopaminergic transmission. Proc. Natl. Acad. Sci. U.S.A., 93, 8716-8720 (1996).

12) Paulson PE, Robinson TE. Relationship between circadian changes in spontaneous motor activity and dorsal versus ventral striatal dopamine neurotransmission assessed with on-line microdialysis. Behav. Neurosci., 108, 624-635 (1994).

13) Ma QP, Yin GF, Ai MK, Han JS. Serotonergic projections from the nucleus raphe dorsalis to the amygdala in the rat. Neurosci. Lett. 134, 21-24 (1991).

14) Härfstrand A, Fuxe K, Cintra A, Agnati LF, Zini I, Wikstrom AC, Okret S, Yu ZY, Goldstein M, Steinbusch H. Glucocorticoid receptor immunoreactivity in monoaminergic neurons of rat brain. Proc. Natl. Acad. Sci. U.S.A., 83, 9779-9783 (1986).

15) Vincent MY, Jacobson L. Glucocorticoid receptor deletion from the dorsal raphé nucleus of mice reduces dysphoria-like behavior and impairs hypothalamic-pituitary-adrenocortical axis feedback inhibition. Eur. J. Neurosci., 39, 1671-1681 (2014).

16) Vincent MY, Donner NC, Smith DG, Lowry CA, Jacobson L. Dorsal raphé nucleus glucocorticoid receptors inhibit tph2 gene expression in male C57BL/6J mice. Neurosci. Lett., 665, 48-53 (2018).

17) Sommer W, Möller C, Wiklund L, Thorsell A, Rimondini R, Nissbrandt H, Heilig M. Local 5,7-dihydroxytryptamine lesions of rat amygdala: release of punished drinking, unaffected plus-maze behavior and ethanol consumption. Neuropsychopharmacology, 24, 430-440 (2001).

18) Takahashi H, Takada Y, Nagai N, Urano T, Takada A. Serotonergic neurons projecting to hippocampus activate locomotion. Brain Res., 869, 194-202 (2000). 